\title{
COMMENT
}

Check for updates

\section{Participant stress in the COVID-19 era and beyond}

Elizabeth V. Goldfarb

The COVID-19 pandemic represents a worldwide stressor. Considering the influence of stress on research participants during this time and beyond may provide new insights and benefit the broader field of human neuroscience.

As human neuroscience researchers, we invite people, with their disparate backgrounds, to participate in experiments to help us uncover fundamental truths about the brain. We are now in the midst of a global pandemic that is changing our lives and the lives of our participants. For those of us who are currently able to work with human participants (in person or online), stress related to the pandemic may have far-reaching and unanticipated effects on the data collected during this period.

Although the pandemic highlights the importance of stress as a factor in everyday life, stress and its neurobiological sequelae are not unique to the pandemic. Indeed, stress can be routinely considered and measured in human neuroscience research. However, outside studies explicitly designed to measure stress or affective processes, stress is not typically considered. Here, I discuss why and how stress should be considered in the design and interpretation of human neuroscience studies during the pandemic and moving forward.

\section{The stress of a pandemic}

Stress is classically described as a response to something novel, unpredictable and uncontrollable - all features of the COVID-19 pandemic. The fear of illness for ourselves and our loved ones for an unknown period of time has been combined with, for example, prolonged disruptions to daily routine, education, child care, occupation and income that have resulted from efforts to limit the spread of the virus ${ }^{1}$. In the United States, these challenges have been disproportionately faced by Black and Indigenous people and other people of colour, who are also systematically exposed to more stressful experiences ${ }^{1,2}$. Isolation and lack of social support can also trigger maladaptive coping behaviours such as increased substance use that can potentiate risk for COVID-19-related complications ${ }^{3}$ and interact with stress pathways to alter brain circuitry and cognitive function ${ }^{4}$.

\section{The impact of stress}

If having participants put their arm in a bucket of icy water for a few minutes (a common laboratory stress-induction protocol) is sufficient to change participants' behaviour and brain responses ${ }^{5}$, one can imagine the scale of the impact that COVID-19-related stress may have on the data being collected now.

Stress leads to changes in neuronal structure and function throughout the brain. These effects are especially pronounced when a stressor is experienced repeatedly or over a long time period, known as chronic stress (which may characterize participants' experiences of the pandemic). Research across different species has shown that chronic stress impairs prefrontal and hippocampal circuits (inducing dendritic atrophy and spine loss), and strengthens responses in the amygdala ${ }^{6,7}$. These changes occur together with effects on myriad cognitive processes, including attention, reward processing, learning, working memory, long-term memory, decision-making, strategy selection and top-down regulation of emotion ${ }^{8}$.

\section{How to respond}

The clinical research community has emphasized the importance of considering the pandemic as a multidimensional stressor to understand mental health consequences $^{1}$. Here, I suggest that this consideration extends beyond clinical science: all the data that are currently being collected from human participants are likely to be influenced by this sustained stress exposure.

When designing studies to examine stress effects, a 'control group' is typically included to match a 'stress group' in as many ways as possible, short of the stress exposure. This enables us to draw conclusions about the specific contributions of stress to the behavioural construct or neural process of interest. However, as practically all humans are now exposed to the pandemic, there is no temporally matched control group (although longitudinal studies may be able to consider pre-pandemic versus post-pandemic differences). Crucially, experiences of and reactions to the challenges associated with the pandemic are highly variable. For example, one participant may have been socially isolated yet had no changes in their employment; another may have grieved the loss of a loved one, taken on new caregiving roles, experienced income loss and increased their alcohol use; and yet another may have had fewer commitments and enjoyed more time with family. 
To better understand human data, and how they may be modulated by stressors like the pandemic, researchers can leverage tools developed by the stress field ${ }^{9}$.

Subjective measures. Several well-validated instruments have been developed to measure the number of stressors people experience and the extent of psychological distress they cause, typically including an assessment of when they occurred during the lifespan ${ }^{10}$. These can be implemented using self-report or interviews by trained personnel. More recently, techniques such as ecological momentary assessment (EMA) use smartphone prompts to track daily fluctuations in experiences of and responses to stressful events. Coping behaviours are also relevant to measure, as some responses, such as escalating alcohol or drug use, can in turn influence behaviour and brain responses ${ }^{4}$. Although self-report measures rely on conscious insight and willingness to describe what may be stigmatized responses, they can help to predict later health outcomes ${ }^{9}$ and can feasibly be incorporated into online and in-person experiments.

Neuroendocrine assays. Testing kits are widely used to measure stress-related hormones in samples of saliva, plasma or hair. Although salivary and plasma cortisol are frequently used to measure acute stress responses in the laboratory, they can also provide insight into more prolonged stress states. For example, basal cortisol levels at different times of day are altered with past stress exposure $^{10}$, stress-related psychopathology ${ }^{8}$ and chronic drug use ${ }^{4}$. Although obtaining saliva and plasma (potentially infectious agents) may be challenging during the pandemic, these measures are routinely used in the stress field, providing a rich comparison data set against which to interpret new data.

Autonomic measures. The body's stress response can also be assessed through measures of autonomic function. Tools to measure autonomic responses include arm cuffs to quantify systolic and diastolic blood pressure, as well as electrocardiograms (ECGs) that can provide ambulatory indices of pulse and heart rate variability. These autonomic functions also adapt with chronic stress and drug use $^{4,9}$. Given the lack of need for bodily fluid samples to assess autonomic responses, such measures would probably be more practicable than neuroendocrine analyses during the pandemic.

Acute stress challenges. Laboratory stressors are frequently used as experimental interventions and can also be used as assays for adaptations in acute stress responses resulting from chronic stress exposure. Participants' reactivity profiles to acute stress can be heightened, blunted or show atypical recovery patterns as a function of their stress histories and biological backgrounds. There are various techniques for inducing stress responses in the laboratory, including physical, psychological and cognitive challenges ${ }^{5}$. By measuring subjective, neuroendocrine and/or autonomic responses before and after such procedures, researchers can quantify acute stress reactivity.
Using stress measures. Beyond studies explicitly designed to examine effects of stress, obtaining stress measures provides opportunities for novel insights into brain and behavioural data. Careful assessment of individuals' stress profiles may enable researchers to account for unanticipated sources of variance. This approach can also allow researchers to interpret individual differences and divide participants into meaningful cohorts, leading to the discovery of novel ways in which neural and behavioural processes are associated with stress.

\section{Looking forward}

The need to consider the affective and physiological context in which people participate in research does not end with the end of COVID-19. We cannot assume that participants always complete our experiments in a neutral state, or that they have comparable histories of stressful experiences. Individual differences in stress and coping might explain hitherto unknown boundary conditions for reproducing effects in human neuroscience, and improve ecological validity by indicating how these processes may occur in the stress-rich context of everyday life. Going forward, participant-level stress profiles can include assessment of remote stress experiences (including the pandemic) as well as in-the-experimental-moment stress reactivity. Broadly considering potential stress effects provides opportunities to identify more brain functions that may be susceptible to stress, elucidate neural factors that determine whether stress is associated with enhanced or impaired function ${ }^{5}$ and uncover protective processes that promote resilience. Knowing what we do about how stress alters fundamental neural processes, examining the effects of the pandemic and other stressors on data from human participants creates an opening to gain crucial insight into the mechanisms of the human brain.

1. Gruber, J. et al. Mental health and clinical psychological science in the time of COVID-19: Challenges, opportunities, and a call to action. Am. Psychol. https://doi.org/10.1037/amp0000707 (2020).

2. Karaca-Mandic, P., Georgiou, A. \& Sen, S. Assessment of COVID-19 hospitalizations by race/ethnicity in 12 states. JAMA Intern. Med. https://doi.org/10.1001/jamainternmed.2020.3857 (2020).

3. Volkow, N. D. Collision of the COVID-19 and addiction epidemics. Ann. Intern. Med. 173, 61-62 (2020).

4. Wemm, S. E. \& Sinha, R. Drug-induced stress responses and addiction risk and relapse. Neurobiol. Stress 10, 100148 (2019).

5. Goldfarb, E. V. Enhancing memory with stress: progress, challenges, and opportunities. Brain Cogn. 133, 94-105 (2019).

6. Arnsten, A. F. Stress weakens prefrontal networks: molecular insults to higher cognition. Nat. Neurosci. 18, 1376-1385 (2015).

7. McEwen, B. S., Nasca, C. \& Gray, J. D. Stress effects on neuronal structure: hippocampus, amygdala, and prefrontal cortex. Neuropsychopharmacology 41, 3-23 (2016).

8. Lupien, S. J., Juster, R. P., Raymond, C. \& Marin, M. F. The effects of chronic stress on the human brain: from neurotoxicity, to vulnerability, to opportunity. Front. Neuroendocrinol. 49, 91-105 (2018).

9. Epel, E. S. et al. More than a feeling: a unified view of stress measurement for population science. Front. Neuroendocrinol. 49, 146-169 (2018)

10. Lupien, S. J., McEwen, B. S., Gunnar, M. R. \& Heim, C. Effects of stress throughout the lifespan on the brain, behaviour and cognition. Nat. Rev. Neurosci. 10, 434-445 (2009).

\section{Acknowledgements}

The author gratefully acknowledges M. D. Rosenberg and D. V. Clewett for helpful discussions.

\section{Competing interests}

The authors declare no competing interests. 